\title{
Accuracy analysis of the GPS instrumental bias estimated from observations in middle and low latitudes
}

\author{
D. H. Zhang ${ }^{1,2}$, W. Zhang ${ }^{1,3}$, Q. Li ${ }^{1,4}$, L. Q. Shi ${ }^{5}$, Y. Q. Hao ${ }^{1}$, and Z. Xiao ${ }^{1}$ \\ ${ }^{1}$ Department of Geophysics, Peking University, Beijing, China \\ ${ }^{2}$ State Key Laboratory of Space Weather, Chinese Academy of Sciences, Beijing, China \\ ${ }^{3}$ Department of Geodesy and Geomatics Engineering, University of New Brunswick, Fredericton, New Brunswick, Canada \\ ${ }^{4}$ Aviation Data and Communication Corporation, Beijing, China \\ ${ }^{5}$ Center for Space Science and Applied Research, China Academic Science, Beijing, China
}

Received: 15 October 2009 - Revised: 4 July 2010 - Accepted: 20 July 2010 - Published: 25 August 2010

\begin{abstract}
With one bias estimation method, the latituderelated error distribution of instrumental biases estimated from the GPS observations in Chinese middle and low latitude region in 2004 is analyzed statistically. It is found that the error of GPS instrumental biases estimated under the assumption of a quiet ionosphere has an increasing tendency with the latitude decreasing. Besides the asymmetrical distribution of the plasmaspheric electron content, the obvious spatial gradient of the ionospheric total electron content (TEC) along the meridional line that related to the Equatorial Ionospheric Anomaly (EIA) is also considered to be responsible for this error increasing. The RMS of satellite instrumental biases estimated from mid-latitude GPS observations in 2004 is around 1 TECU $\left(1 \mathrm{TECU}=10^{16} / \mathrm{m}^{2}\right)$, and the RMS of the receiver's is around 2TECU. Nevertheless, the RMS of satellite instrumental biases estimated from GPS observations near the EIA region is around 2 TECU, and the RMS of the receiver's is around 3-4 TECU. The results demonstrate that the accuracy of the instrumental bias estimated using ionospheric condition is related to the receiver's latitude with which ionosphere behaves a little differently. For the study of ionospheric morphology using the TEC derived from GPS data, in particular for the study of the weak ionospheric disturbance during some special geo-related natural hazards, such as the earthquake and severe meteorological disasters, the difference in the TEC accuracy over different latitude regions should be paid much attention.
\end{abstract}

Keywords. Ionosphere (Equatorial ionosphere)

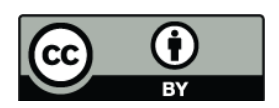

Correspondence to: D. H. Zhang

(zhangdh@pku.edu.cn)

\section{Introduction}

The capability of dual carrier frequencies of GPS system to remove ionospheric delays provides a useful tool for measuring the ionospheric total electron content (TEC) (Lanyi and Roth, 1988; Coco et al., 1991). With the wide application of high-precision GPS technology, the GPS receiver has gradually become a routine and effective equipment for ionospheric measurements. The advantages of globally distributed receivers and easy data availability make the collection of the ionospheric TEC derived from raw GPS observations very convenient. Actually, like the ionospheric F layer critical frequency, foF2, obtained using ionosonde, the ionospheric TEC already becomes a basic ionospheric parameter to describe ionospheric behavior. Internet-shared and globally distributed GPS observations provide the worldwide researchers with a facility to study the local, regional and global ionosphere independently, quickly and simultaneously. Research on ionospheric morphology and disturbance based on this parameter greatly extends our understanding for the ionospheric temporal and spatial variations (Ho et al., 1996; Mannucci et al., 1998; Mendillo, 2000; Zhang and Xiao, 2005).

In the initial study of the ionospheric TEC using GPS method, the accuracy and the stability of the instrumental bias estimated from GPS observations was one of the key points of interest. The evaluating studies between TEC from GPS data and that from other scientific instruments were reported in a number of papers (Brunini et al., 2005; Ho et al., 1997; Vladimer et al., 1997). It is found that the GPS instrumental biases are the main error source in the process of acquiring ionospheric TEC from GPS observations, and sometimes the value of the instrumental biases is even larger than

Published by Copernicus Publications on behalf of the European Geosciences Union. 
that of the GPS signal delays caused by background ionosphere. Therefore, only under the correct estimation of the instrumental biases, the analysis results of the ionospheric morphology based on TEC derived from GPS observations are believable. Nevertheless, as for most GPS users, it is very difficult or sometimes impossible to measure GPS instrumental biases in real-time during the period of GPS operation. In order to derive the correct ionospheric TEC from raw GPS observations, a number of methods to estimate instrumental biases have been developed under some assumptions on ionospheric condition. To convert a slant path TEC to a vertical TEC, the ionosphere is assumed to be a thin shell encircling the Earth with an average ionospheric height that is usually set as constant value during TEC calculation from GPS observation. This assumption undoubtedly introduces error during the derivation process of vertical ionospheric TEC from GPS data. Another basic ionospheric condition assumes the ionospheric variation stable in the sun-fixed coordinate system, or the ionosphere should exhibit smooth temporal and spatial variation (Sardon et al., 1994; Sardon and Zarraoa, 1997; Ciraolo et al., 2007; Ma and Maruyama, 2003; Lunt et al., 1999). This assumption is satisfied in most situations, but in some special ionospheric conditions or in some special ionospheric regions, the error of this ionospheric assumption is larger. Actually, some kind of severe disturbing phenomena can make the ionosphere deviate the average ionospheric status greatly. These disturbances can degrade the accuracy of the estimated instrumental biases and ionospheric TEC from GPS data based on the smooth ionospheric condition.

Ionospheric storm is one of the important ionospheric disturbing phenomena. During storm period, the ionosphere exhibits severe deviation from background ionosphere that lasts from several hours to several days and covers regionally or globally. Based on the GPS data observed in mid-latitude region from 2004 to 2006, the instrumental bias were obtained using a estimation method of instrumental bias, and the differences of the instrumental bias between the active geomagnetic days and the quiet geomagnetic days were studied (Zhang et al., 2009a). It is found that the standard deviation of instrumental biases during active geomagnetic days is obviously larger than that during the quiet days, and this deviation can degrade the accuracy of ionospheric TEC derived from GPS observations.

The significant effect of the asymmetrical distribution of the plasmaspheric electron content on the GPS instrumental bias estimation has been studied, and it is found that the accuracy of ionospheric TEC increases if considering the plasmaspheric electron content meridional distribution during bias estimation (Lunt et al., 1999; Mazzella et al., 2002). Besides the latitude-related distribution of the plasmasphere, the ionospheric electron content also exhibits an obvious asymmetrical distribution along the meridional line related to the Equatorial Ionospheric Anomaly (EIA) that strongly controls the variation of the low latitude ionosphere. Comparing with
Table 1. Station ID, receiver types, geographic and geomagnetic latitude (lat) and longitude (lon) used in the paper.

\begin{tabular}{|c|c|c|c|c|c|}
\hline \multirow{2}{*}{$\begin{array}{l}\text { Station } \\
\text { ID }\end{array}$} & \multirow[t]{2}{*}{ Receiver type } & \multicolumn{2}{|c|}{ Geographic } & \multicolumn{2}{|c|}{ Geomagnetic } \\
\hline & & lat & lon & lat & lon \\
\hline GUAO & Ashtech UZ-12 & 43.47 & 87.18 & 34.04 & 162.05 \\
\hline BJFS & Ashtech Z-XII3 & 39.60 & 115.89 & 29.7 & 187.19 \\
\hline XNIN & Ashtech Z-XII3 & 36.60 & 101.77 & 26.66 & 174.60 \\
\hline TAIN & Ashtech Z-XII3 & 36.22 & 117.12 & 26.35 & 188.40 \\
\hline XIAA & Ashtech Z-XII3 & 34.18 & 108.98 & 24.20 & 181.09 \\
\hline WUHN & Ashtech Z-XII3 & 30.53 & 114.36 & 20.60 & 186.05 \\
\hline LUZH & Ashtech Z-XII3 & 28.87 & 105.41 & 18.89 & 177.81 \\
\hline XIAG & Ashtech Z-XII3 & 25.61 & 100.25 & 15.71 & 172.95 \\
\hline XIAM & Ashtech Z-XII3 & 24.45 & 118.08 & 14.62 & 189.68 \\
\hline GUAN & Ashtech Z-XII3 & 23.19 & 113.34 & 13.25 & 185.25 \\
\hline QION & Ashtech Z-XII3 & 19.03 & 109.84 & 9.05 & 181.97 \\
\hline
\end{tabular}

the mid-latitude ionosphere, the low latitude ionosphere exhibits greater spatial gradient and more intensive temporal variation, so it is more difficult to describe the ionospheric variations in this region. Therefore, the accuracy of GPS instrumental biases estimated from low latitude GPS observation based on the ionospheric model should be lower than that estimated from the mid-latitude data. Undoubtedly, a detailed quantitative analysis about the instrumental bias accuracy is useful for the correctly understanding of the ionospheric TEC results. Recently, some accuracy analysis for the ionospheric TEC estimated from GPS observations over low latitudinal region has been reported (Niranjan et al., 2007; Mazzella et al., 2007; Thampi et al., 2007). In this paper, using the observations of the GPS receivers located in the middle and low latitude in China longitude sector in 2004, the statistical analysis for the instrumental biases estimated in different latitude will be studied. Additionally, the error range of the ionospheric TEC derived from GPS observations in different latitude will be estimated based on the variation of the instrumental bias.

\section{Data and method for the determination of GPS instrumental biases}

Considering the possible influence of the ionospheric variability during different solar cycle period on the accuracy of the instrumental bias estimation, the GPS data in solar minimum year (2004) are selected. All the GPS stations are located in China longitude sector and in middle and low latitude. The GPS data are in RINEX format, and the sampling rate is $30 \mathrm{~s}$. The GPS station ID, the type of the receivers, and the position of the stations are shown in Table 1.

The measurements of the pseudo-ranges and carrier phases in two L-band carrier frequencies $\left(f_{\mathrm{L} 1}=1575.42 \mathrm{MHz}\right.$, $\left.f_{\mathrm{L} 2}=1227.60 \mathrm{MHz}\right)$ are included in GPS data. The absolute TEC (Slant_TEC ${ }^{p}$ ) and relative TEC (Slant_TEC ${ }^{l}$ ) along the line from satellite to receiver can be calculated using 
following equations, respectively (Mannucci et al., 1998; Ma and Maruyama, 2003):

Slant_TEC ${ }^{p}=\frac{2\left(f_{1} f_{2}\right)^{2}}{k\left(f_{1}^{2}-f_{2}^{2}\right)}\left(\mathrm{P}_{2}-\mathrm{P}_{1}\right)$

Slant_TEC ${ }^{l}=\frac{2\left(f_{1} f_{2}\right)^{2}}{k\left(f_{1}^{2}-f_{2}^{2}\right)}\left(\mathrm{L}_{1} \lambda_{1}-\mathrm{L}_{2} \lambda_{2}\right)$

where $k=80.62 \mathrm{~m}^{3} \mathrm{~s}^{-2}, \lambda_{1}$ and $\lambda_{2}$ are the wavelength corresponding to $\mathrm{f}_{L 1}, \mathrm{f}_{L 2}, \mathrm{P}_{1}$ and $\mathrm{P}_{2}$ are the pseudo-range measurments, and $L_{1}$ and $L_{2}$ are the carrier phase measurments at $\mathrm{f}_{L 1}, \mathrm{f}_{L 2}$. Because of the potential ambiguity in the carrier phase measurement, the Slant_TEC ${ }^{l}$ in Eq. (2) is only a relative slant TEC value. However, the relative error of the Slant_TEC ${ }^{l}$ is much lower than that of the absolute TEC obtained from Eq. (1). In the actual application, combining the Slant_TEC ${ }^{l}$ and the Slant_TEC ${ }^{p}$ during one continuous tracking arc, the offset value $\mathrm{B}_{\mathrm{rs}}$ between the Slant_TEC ${ }^{l}$ and the Slant_TEC ${ }^{p}$ can be calculated using Eq. (3), and the absolute slant TEC with higher accuracy in each observation epoch can be obtained from Eq. (4) (Mannucci et al., 1998; Ma and Maruyama, 2003).

$B_{\mathrm{rs}}=\frac{\sum_{k=1}^{N}\left(\text { Slant_TEC }_{k}^{p}-\text { Slant_TEC }_{k}^{l}\right) \sin ^{2} \mathrm{el}_{k}}{\sum_{k=1}^{N} \sin ^{2} \mathrm{el}_{k}}$

Slant_TEC $=$ Slant_TEC ${ }^{l}+B_{\mathrm{rs}}$

where $N$ is the number of observation epochs during a continuous tracking arc, $\mathrm{el}_{k}$ is the satellite's elevation angle, and the term $\sin ^{2} \mathrm{el}_{k}$ is a weighting factor for restraining the socalled signal multipath effect. As the pseudo-range with low elevation angle is apt to be affected by the multipath effect and the reliability decreases, consequently, the contribution to the $B_{\mathrm{rs}}$ determination is greatly diminished from slant paths with low elevations in Eq. (3). Thus, the absolute slant TECs, which are free of ambiguities and have low noise and multipath effects, can be obtained from Eq. (4) (Mannucci et al., 1998; Ma and Maruyama, 2003). In this study, the values are restricted to satellite elevation angles larger than $30^{\circ}$. Considering the satellite and receiver instrumental biases, the vertical ionospheric TEC at the ionosphere pierce point (IPP) (the intersecting point between the line from the satellite to the receiver and the ionosphere shell) can be obtained by using the following relation:

$\mathrm{TEC}=\left(\right.$ Slant $\_$TEC $\left.-B_{\mathrm{S}}-B_{\mathrm{r}}\right) \cos E_{\mathrm{ion}}$

where $B_{\mathrm{s}}$ and $B_{\mathrm{r}}$ are the satellite and receiver instrumental biases, respectively. $E_{\text {ion }}$ is the mapping angle that can be obtained from Eq. (6):

$E_{\text {ion }}=\arcsin \left(\frac{R_{\mathrm{E}}}{R_{\mathrm{E}}+H_{\mathrm{i}}} \cos \mathrm{el}\right)$ where $R_{\mathrm{E}}$ is the mean radius of the Earth and $H_{\mathrm{i}}$ is the height of the ionospheric shell (assumed to be $400 \mathrm{~km}$ in this study).

The instrumental biases (including satellite and receiver) and vertical TEC in Eq. (5) can be determined using the selfcalibration of pseudo-range errors (SCORE) process (Lunt et al., 1999). The basic concept in the SCORE process is that when the IPP-derived line between two satellites and a receiver arrive at the same moment at a same point, that is, when a so-called conjunction occurs, the vertical TECs at the two IPP derived using Eq. (5) are assumed equality. It should be noted that this concept implies an ionospheric background model that the ionosphere over the same latitudinal zone has the same diurnal variation, or, the concept contains an assumption of the ionospheric condition with smooth spatial and temporal variation. For a certain data sample, assuming Slant_TEC $\alpha\left(\theta_{\alpha}, \tau_{\alpha}\right)$ and Slant_TEC $\beta\left(\theta_{\beta}, \tau_{\beta}\right)$ are the slant TECs at the two IPPs of the line of the receiver and GPS satellites marked $\alpha$ and $\beta$, using the assumption outlined above, when a conjunction occurs at the two IPPs, we obtain the following equation:

(Slant_TEC $\alpha-B_{\alpha}-B_{\mathrm{r}}$ ) $\cos E_{\text {ion } \alpha}$

$=\left(\right.$ Slant $\left._{-} \mathrm{TEC}_{\beta}-B_{\beta}-B_{\mathrm{r}}\right) \cos E_{\mathrm{ion} \beta}$

In our calculation, the ionospheric shell is divided into even meshes of 0.5 degrees by $0.1 \mathrm{~h}$ in latitude and local time, respectively. For each conjunction pair, the satellite and receiver pair in the same mesh can be expressed in the form of Eq. (7). Thus, we obtain a set of overdetermined linear equations with the instrumental biases as unknowns that are written in matrix form in Eq. (8).

$$
\begin{aligned}
& {\left[\begin{array}{ccccc}
\cdots & \cdot & \cdots & . & \cdots \\
\cdots & \cdot & \cdots & \cdot & \cdots \\
\cdots & \cos E_{\mathrm{ion} \beta i} & \cdots & -\cos E_{\mathrm{ion} \alpha i} & \cdots \\
\cdots & \cdot & \cdots & \cdot & \cdots \\
\cdots & \cdot & \cdots & . & \cdots
\end{array}\right]\left[\begin{array}{c}
B_{\mathrm{s}=1}+B_{\mathrm{r}} \\
\vdots \\
\vdots \\
\vdots \\
\vdots \\
B_{\mathrm{s}=32}+B_{\mathrm{r}}
\end{array}\right]} \\
& =\left[\begin{array}{c}
\cdots \\
\cdots \\
\text { Slant_TEC }{ }_{\beta i} \cos E_{\text {ion } \beta i}-\text { Slant_TEC }_{\alpha i} \cos E_{\text {ion } \alpha i} \\
\cdots \\
\cdots
\end{array}\right]
\end{aligned}
$$

For one day's GPS observations in $30 \mathrm{~s}$ sampling rate over Chinese region, the number of the equation in Eq. (8) is about 200 300. Assuming the instrumental bias unchangeable during one day's period, the daily GPS instrumental biases $\left(B_{\mathrm{S}}+B_{\mathrm{r}}\right)$ can be obtained through solving Eq. (8) using a least-squares method. In addition, under the zero-mean condition imposed on the estimated satellites instrumental bias, the receiver's instrumental bias can be obtained simultaneously. It should be noted that, in this study, the ionospheric 
Table 2. The RMS of the satellite instrumental bias obtained from CODE and estimated using SCORE method based GUAO data over 2004 (unit: TECU).

\begin{tabular}{lcccccccccccccc}
\hline PRN & 1 & 3 & 4 & 5 & 6 & 7 & 8 & 9 & 10 & 11 & 13 & 14 & 15 & 16 \\
CODE(rms) & 0.87 & 0.86 & 0.89 & 0.84 & 0.84 & 0.68 & 1.27 & 0.73 & 0.78 & 0.93 & 0.84 & 0.92 & 0.88 & 0.87 \\
GUAO(rms) & 0.77 & 0.91 & 0.74 & 0.86 & 0.62 & 0.66 & 0.96 & 0.56 & 0.65 & 1.08 & 0.87 & 0.62 & 0.49 & 0.81 \\
PRN & 17 & 18 & 19 & 20 & 21 & 22 & 24 & 25 & 26 & 27 & 28 & 29 & 30 & 31 \\
CODE(rms) & 0.73 & 0.76 & 0.67 & 0.89 & 0.78 & 0.84 & 0.81 & 0.82 & 0.75 & 0.82 & 0.89 & 0.78 & 0.80 & 0.89 \\
GUAO(rms) & 0.65 & 0.44 & 0.68 & 0.97 & 0.47 & 0.50 & 0.68 & 0.50 & 0.52 & 0.96 & 0.64 & 0.67 & 0.60 & 0.69 \\
\hline
\end{tabular}

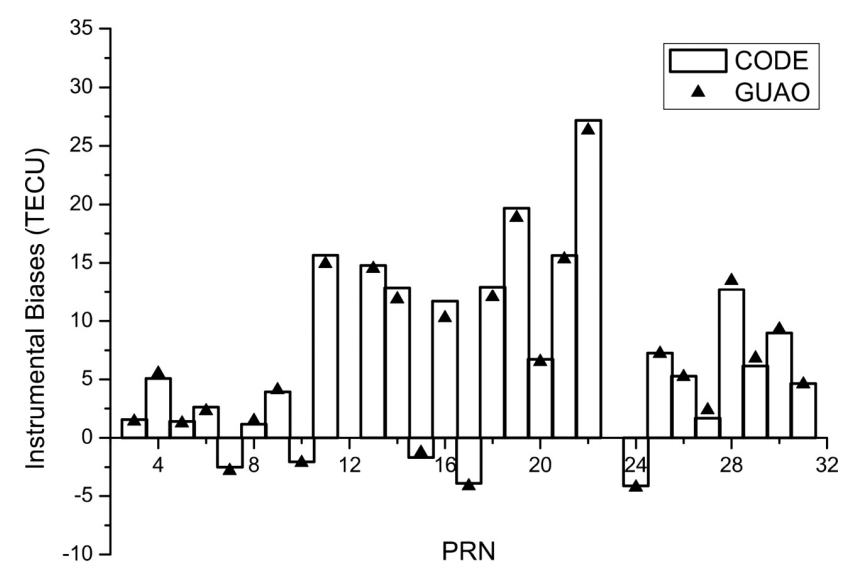

Fig. 1. Comparison of the instrumental biases issued by CODE with those estimated from GUAO data using the described method in 2004.

shell is divided into even meshes of 0.5 degrees by $0.1 \mathrm{~h}$ in latitude and local time, the ionospheric correlation region is limited to each little mesh area. The Eq. (7) is obtained only under the condition of the "conjunctions" occurring in the same mesh. So the function to weight the measurement contribution in different temporal and spatial scale over whole ionospheric shell used in the original SCORE method is not introduced here.

In this paper, for the convenience to analyze the influence of the instrumental biases on the accuracy of ionospheric TEC, the unit of instrumental bias is transferred as TECU, where 1 nanosecond (ns) is equivalent to 2.853 TECU $\left(1 \mathrm{TECU}=10^{16} / \mathrm{m}^{2}\right)$. Figure 1 shows the mean values of GPS satellite instrumental biases in 2004 estimated from GUAO observations using the method introduced above (relative to the instrumental bias of PRN 1). The results of instrumental biases issued by CODE are also shown in this figure, and the corresponding RMS of the biases in Fig. 1 is also given in Table 2. (The result of satellite PRN 2 and PRN 23 is not included due to significant satellite bias changes associated with equipment activation in 2004.) It can be seen that the accuracy of the two methods are very close.

\section{Results and analysis}

Figure 2 shows the instrumental biases of several GPS satellites estimated from the GUAO station in 2004 relative to the mean value of the whole year. The $\mathrm{X}$-axis denotes the day of year (DOY). In order to compare the difference with the results of the low latitude station clearly, the Y-axis range is set from -10 to 10 TECU for all listed satellites. For the PRN 19 satellite, there is a data gap before March 2004 due to the unavailability of the satellite. Figure 3 shows the satellites' instrumental biases based on the data of the XIAM station relative to the mean value of the whole year in 2004. From Figs. 2 and 3, which are the results from the typical middle and low latitude stations, it can be seen that the instrumental biases estimated from the mid-latitude GUAO observations had small fluctuations throughout 2004, and nearly all of the instrumental biases appear in the range of $-2 \sim 2$ TECU. The RMS values of instrumental biases throughout 2004 for all listed satellites are less than 1 TECU. Nevertheless, the satellite instrumental biases estimated from the XIAM observations, which is located in the low latitude region, fluctuate much more extensively than that estimated from GUAO observations. The value between the maximum and minimum of instrumental biases for some satellites estimated from XIAM observations even reaches more than 15 TECU. In addition, the RMS values of the satellites for the whole year are obviously higher than the results estimated from the GUAO data, but usually less than 2.5 TECU.

From the results above, it can be seen that the accuracies of satellite instrumental biases derived from the GPS observations at low and middle latitude using the same estimating method are very different. The instrumental biases estimated using the same method exhibit much more fluctuations in the low latitude regions. Considering the estimating process of the instrumental bias introducing above, it is reasonable to deduce that this difference is mainly caused by the different ionospheric morphology in the middle and low latitude region. In order to show the tendency of the estimated instrumental biases with latitude further, Fig. 4 gives the RMS values of the instrumental biases of 10 GPS satellites estimated from the data of the stations in 2004 listed in Table 1. The labels on the $\mathrm{X}$-axis are the station names, which are arranged in the order from higher latitude to lower latitude. The Y-axis denotes the RMS values of the satellite instrumental biases 

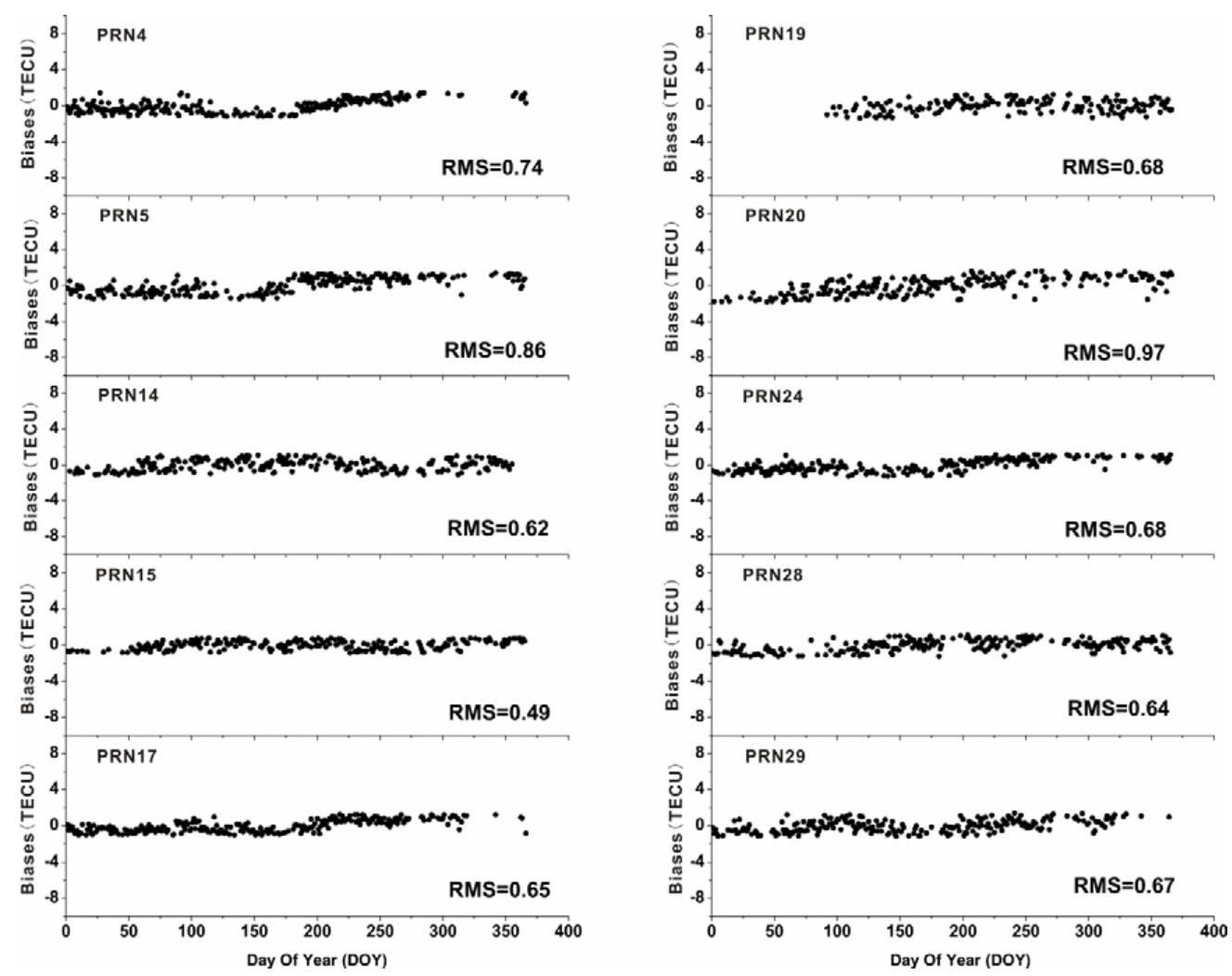

Fig. 2. Satellite instrumental biases estimated from GPS data observed at GUAO station in 2004 (relative to the mean value).
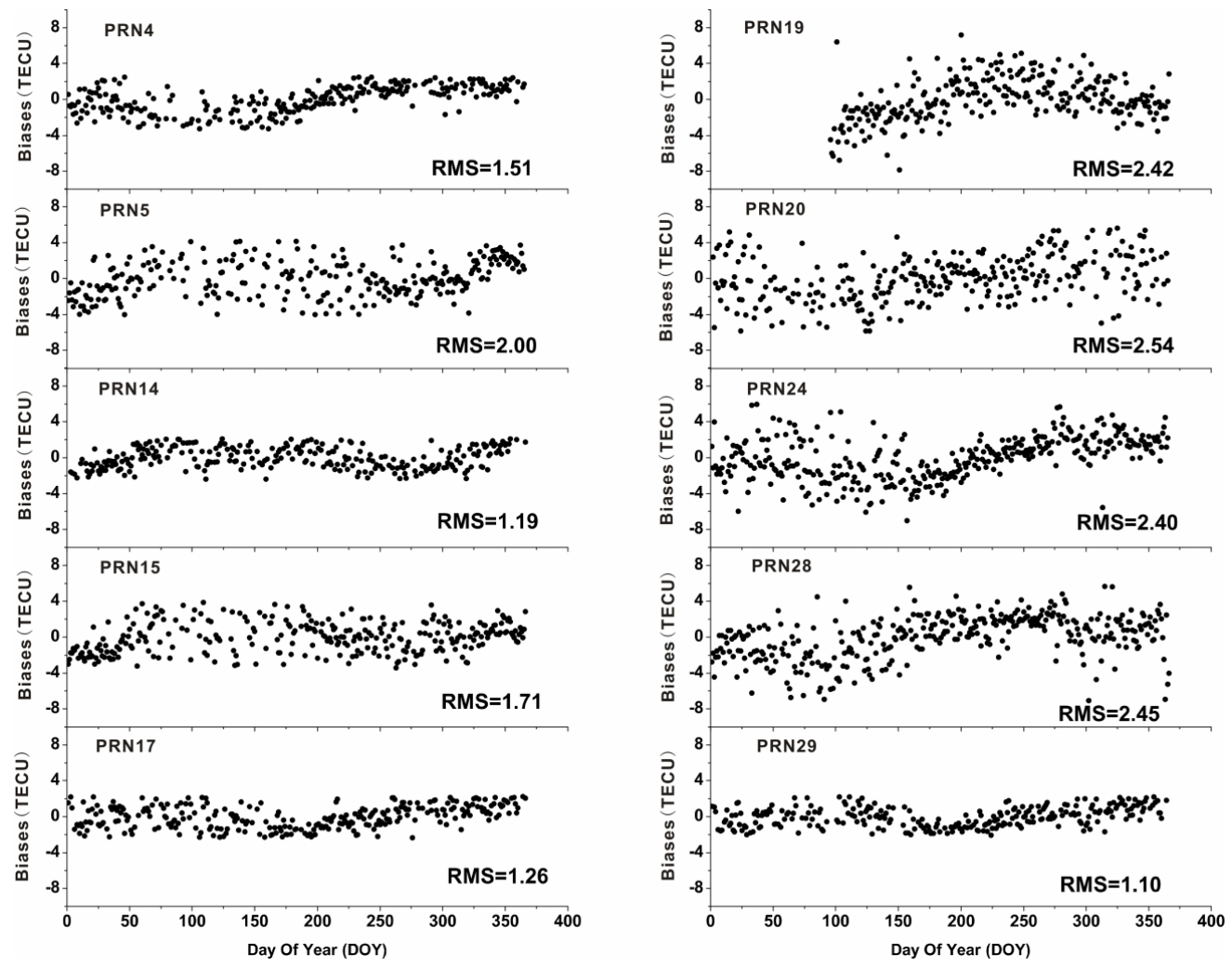

Fig. 3. Satellite instrumental biases estimated from GPS data observed at XIAM station in 2004 (relative to the mean value). 

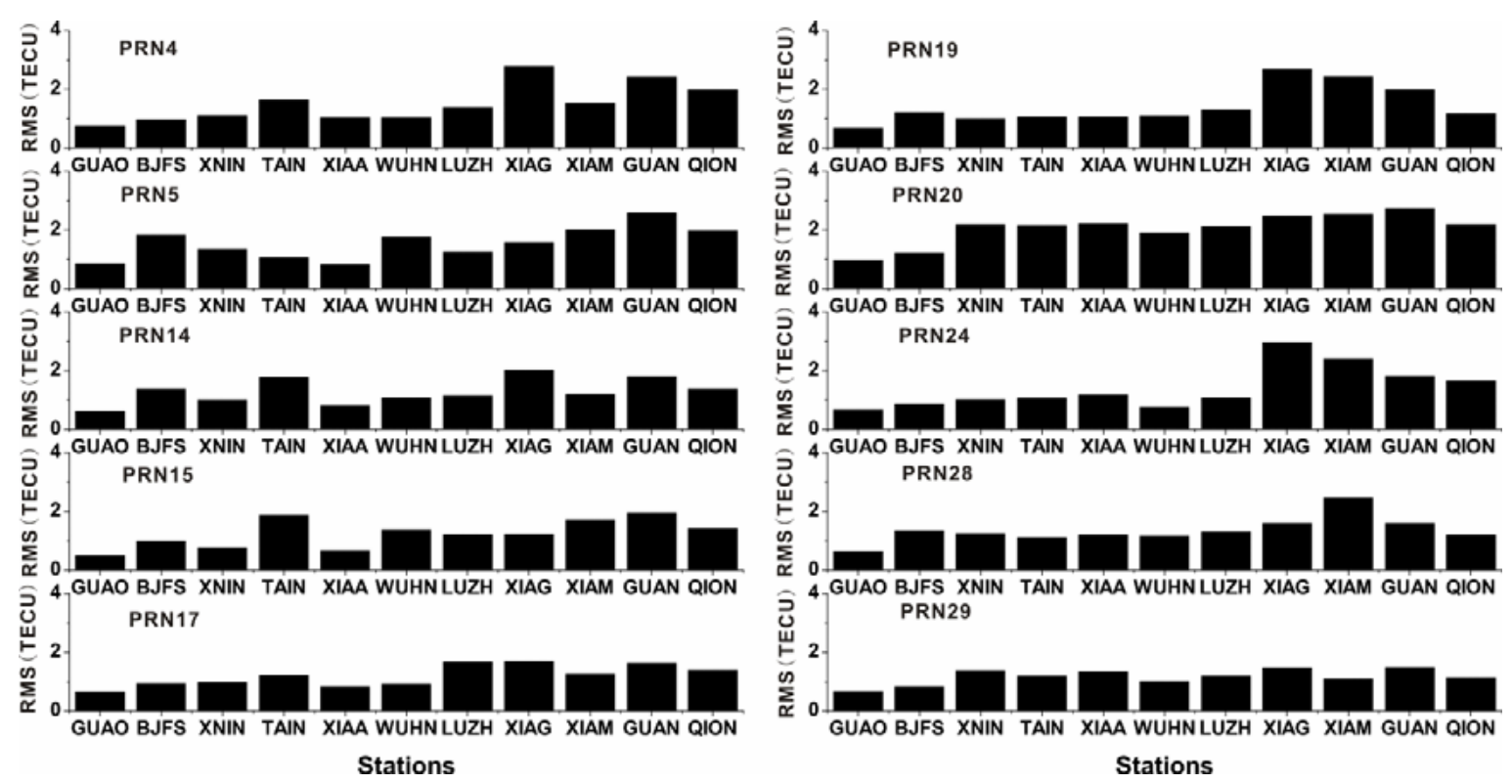

Fig. 4. RMS of instrumental biases of ten GPS satellites estimated from GPS data in 2004.

estimated from the GPS observations in 2004. From Fig. 4, it can be seen that, although there are a little exceptional situations, the main tendency of the RMS value of the satellite instrumental bias is that the RMS values estimated from the data observed in the low latitude region is larger than that estimated from the data in the middle latitude. The RMS value varies from approximately 1 to 3 TECU, but all RMS values are less than 4 TECU. Furthermore, it can be seen that the RMS values of the satellite instrumental biases estimated from the QION station, located at the southern side of the equatorial anomaly north peak, are less than the RMS estimated from the XIAG, GUAN, and XIAM stations located just below the EIA crest. Certainly, the dependence of RMS on latitude does not show very obviously in the results estimated from the data of these stations located in the transitional region between middle and low latitude. The RMS of some satellites instrumental bias estimated from the BJFS and TAIN data seems larger than the other stations'. The data quality analysis using the occurrence of GPS cycle slip derived from the GPS data observed at two nearby sites reveals the existence of the data quality differences (Zhang et al., 2010). Even for the same type of the receiver and antenna, the so-called inter-individual differences of the observations exist due to the receivers' hardware thermal noise conditions and GPS sites nearby environment. Although some measures are taken to alleviate the possible influence on the estimation of the GPS instrumental bias, the inter-individual differences of the receivers still exist. The larger RMS of the bias estimated from BJFS and TIAN observation maybe illustrate that the inter-individual differences of the equipments and the observing environment can affect the accuracy of the bias estimation. Even so, the main tendency of the latitude varying RMS is explicit in Fig. 4.

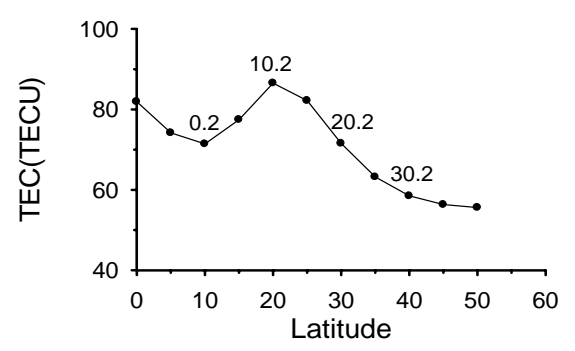

Fig. 5. The geographical latitudinal distribution (from $0^{\circ} \mathrm{N}$ to $50^{\circ} \mathrm{N}$ ) of TEC along the $120^{\circ}$ meridional line calculated from IRI model (IRI model parameters: longitude: $120^{\circ} \mathrm{E}$; day of year: 280 ; local time: $12 \mathrm{LT}$; R12: 150; height range selection: $100-1000 \mathrm{~km}$; height step: $1 \mathrm{~km}$ ). The values labeled in the figure are the corresponding geomagnetic latitudes.

Other research has found that the distribution of the plasmaspheric electron content along the meridional line has a significant effect on TEC and GPS instrumental bias estimation, and the accuracy of the instrumental bias can be improved by properly considering the distribution of the plasmaspheric electron content (Lunt et al., 1999; Mazzella et al., 2002, 2007, 2009; Anghel et al., 2009; Carrano et al., 2009). Based on this improvement the distribution of the plasmaspheric electron content was estimated using GPS measurements (Mazzella et al., 2002, 2007; Anghel et al., 2009; Carrano et al., 2009). Besides the latitude-related distribution of the plasmasphere, the ionosphere also exhibits an obvious latitude distribution near the EIA region (the geomagnetic latitude of peak EIA is about $10^{\circ}-15^{\circ}$ ) that is mainly controlled by the ionospheric $\mathrm{E}$ region electric field (Kelley and Heelis, 1989). In Chinese longitudal sector, the influence of the EIA can reach the region of the $40^{\circ} \mathrm{N}$ (about $30^{\circ}$ geomagnetic latitude). Figure 5 gives the geographical 

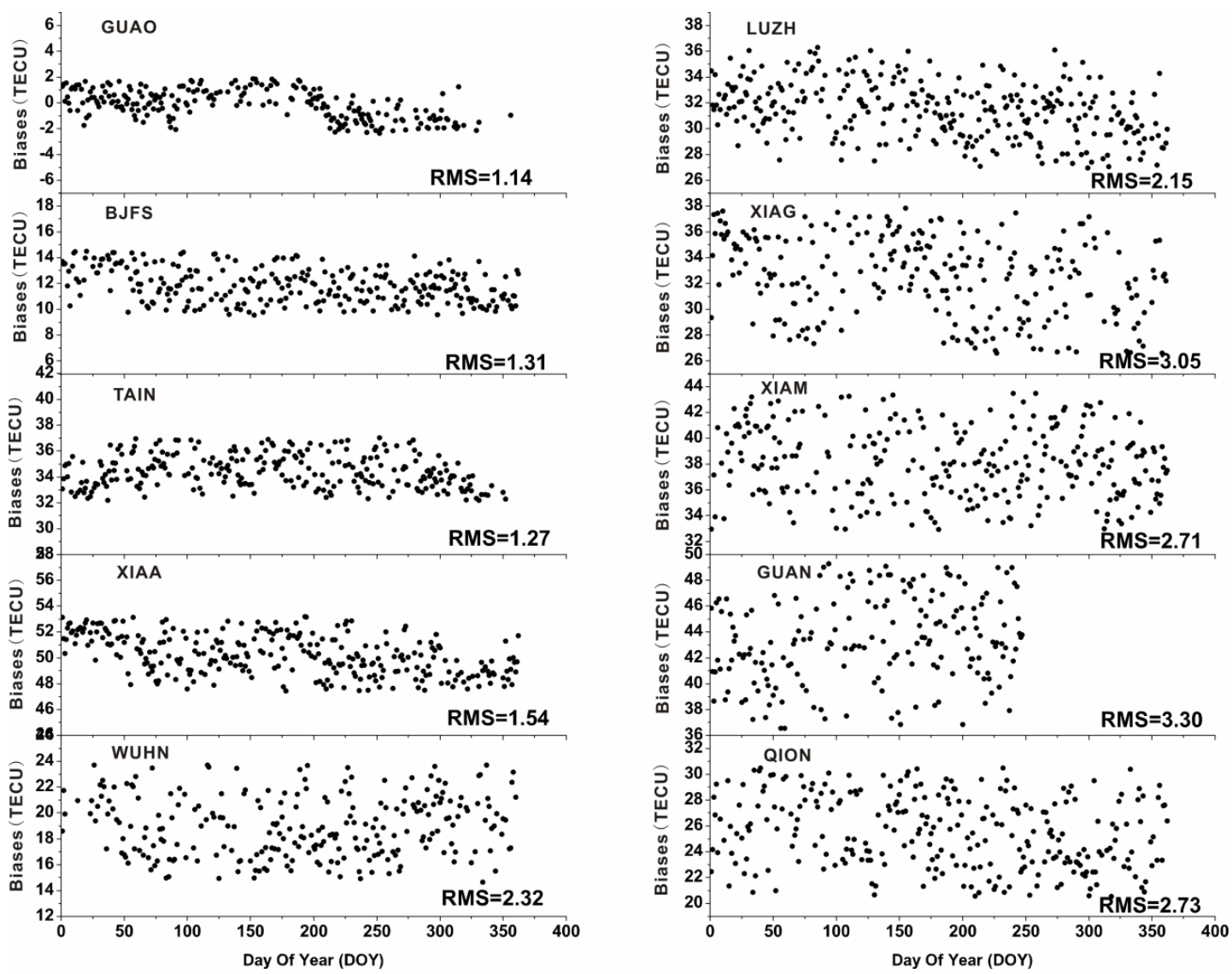

Fig. 6. Receiver instrumental biases estimated from GPS data in 2004.

latitudinal distribution (from $0^{\circ} \mathrm{N}$ to $50^{\circ} \mathrm{N}$ ) of the ionospheric TEC along $120^{\circ} \mathrm{E}$ meridional line obtained by integrating ionospheric electron content from the height of $100 \mathrm{~km}$ to $1000 \mathrm{~km}$ that is calculated from International Reference Ionosphere model (IRI) in solar maximum phase. The values labeled in the figure are the corresponding geomagnetic latitudes. The distribution of northern EIA can be seen obviously in this figure. With the latitude decreasing, the ionospheric spatial gradient in the meridian direction induced by the northern EIA increases gradually. It is observed that in solar maximum years, in the longitude sector of China in the latitude range from $30^{\circ} \mathrm{N}$ to $35^{\circ} \mathrm{N}$, the gradient of ionospheric TEC in the meridian direction induced by equatorial anomaly reaches around 2 TECU/degree, and it becomes larger with latitude decreasing (Zhang et al., 2002). On the other hand, comparing with the mid-latitude ionosphere, the low latitude ionosphere exhibits more significant and complicated temporal variability due to the electro-dynamic process occurred in the ionospheric $\mathrm{E}$ and $\mathrm{F}$ region. Therefore, during the process of estimating instrumental bias, with the latitude decreasing, the error of the assumption, which the vertical ionospheric TECs at the different IPP but in a same divided mesh are equal (described in Eq. 7), becomes larger gradually due to significant spatial and temporal variations.
Then, when solving Eq. (8) using least-square method, the standard deviation of the solutions using low latitude observations should be larger than that of the solutions estimated from mid-latitude observations. This cause the RMS of the estimated instrumental bias becoming large. This may be the one of main reasons for why the RMS of instrumental biases shown in Fig. 4 gradually increases and the accuracy becomes worse with latitude decreasing. Certainly, the influence of the distribution of the plasmasphere along the meridional line in the lower latitude on the estimation of the instrumental bias should also be noted (Lunt et al., 1999; Mazzella et al., 2007). Although this brings about the complication for the interpretation of these results, further study to distinguish their influences can increase undoubtedly the accuracy of the ionospheric and plasmaspheric TEC from GPS observations.

Under the zero-mean condition imposed on the estimated satellites instrumental bias, the receiver's instrumental bias can be obtained. Figure 6 shows the receivers instrumental biases estimated from GPS observations of 10 stations in 2004. Different from the satellite instrumental bias in Figs. 2 and 3, the Y-axis in this figure denotes the absolute value of receiver instrumental bias. Because the GPS receiver in GUAN station was replaced with a new receiver after DOY 248 in 2004, the receiver instrumental biases after 


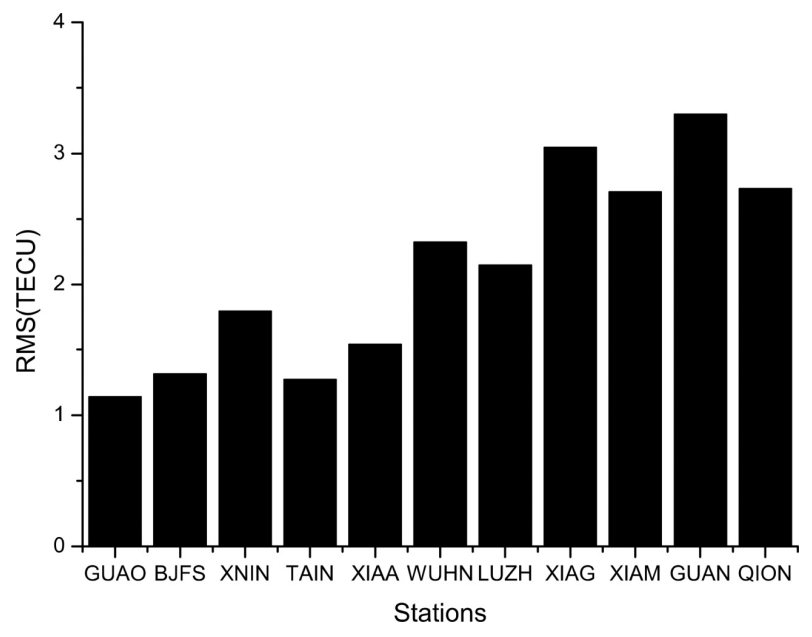

Fig. 7. RMS of the receivers' instrumental biases estimated from GPS data in 2004.

this day are not given in this figure and the RMS value is calculated just based on the data before DOY 248. It can be seen in Fig. 6 that the scatter range of the instrumental bias estimated from the GPS data is different and the RMS value of the receiver instrumental bias located in mid-latitude is less than that in low latitude. In order to show this feature clearly, the RMS values of the receiver instrumental bias is given in Fig. 7 listed in the order of latitude. It can be seen that the RMS of instrumental bias increases with the latitude decreasing roughly, and the RMS value changes from about 1 TECU to 4 TECU.

The largest error source of the ionospheric TEC derived from GPS method can be the error of the measured or estimated instrumental bias. To a certain extent, the accuracy of the estimated instrumental bias determines the accuracy of TEC. From the RMS variation of the satellite and receiver instrumental bias corresponding to the latitudes mentioned above, it can be concluded that, besides the influence of the variability of the plasmasphere, the equatorial ionospheric anomaly that deviates the assumptions of ionospheric smooth variation and fixed ionospheric shell height also affect the accuracy of the bias estimation. The accuracy of TEC derived from stations located in the mid-latitude region is higher than that in low latitude. Considering the error of both GPS satellite and receiver instrumental biases based on the ionospheric model assumption, the accuracy of ionospheric absolute TEC derived from GPS data in the mid-latitude region using this method is around 2-4 TECU, whereas that in the low latitude region is around 6-8 TECU.

Besides the SCORE method, many advanced dataprocessing technologies and methods, such as neural networks (Ma et al., 2005), Kalman filter (Sardon et al., 1994; Anghel et al., 2009; Carrano et al., 2009), SCORPION method that removes the contribution of the plasmasphere using some model (Mazzella et al., 2002, 2007), have been used in the process to estimate the instrumental biases in the last decade. Nevertheless, no matter which methods are employed, the condition of the temporal and spatial variation of the ionosphere has to be considered during the process to estimate the GPS instrumental biases from raw GPS data, and the large horizontal gradient of the ionospheric TEC caused by the EIA certainly will affect the accuracy of the estimated bias. Although the receiver bias can be measured using some special equipments, such as GPS receiver calibrator (Dyrud et al., 2008), obtaining the instrumental bias independently of ionospheric condition is still impractical for most GPS users. Nevertheless, ionosphere exhibits temporal and spatial variability, and up to now the accurate ionospheric model to describe this variability is unobtainable. So, the influence of the ionospheric variability on the accuracy of the instrumental bias estimation is difficult to remove completely. In the ionospheric morphological studies, the researchers should be aware of the errors. The advent of the GPS greatly stimulates the study of the ionospheric morphology and disturbance. Apart from traditional ionospheric study (Ho et al., 1996; Mendillo, 2006), the ionospheric disturbances related with some geo-related natural hazards, such as the great earthquakes or severe meteorological disasters, also draw attentions from the global geophysicists (Liu et al., 2004; Wan et al., 1998; Xu et al., 2008). The spatial scale of the ionospheric disturbances caused by these kinds of events generally covers hundreds or thousands of kilometers, and the variation of the ionospheric TEC disturbance is usually several TECU that is much less than that of the ionospheric storm. Therefore, during the process to derive the ionospheric TEC from the GPS data, the TEC error problem related to instrumental bias should be noted. That is, attention should be paid to the results based on the ionospheric TEC derived from GPS data, and the reliability analysis is necessary for the study of the weak TEC disturbances that are less than or similar to the error scale of the TEC mentioned above.

The ionospheric spatial and temporal variation depends on the solar cycle, and the spatial gradient during solar minimum period is much less than that during solar maximum period. Therefore, the RMS of the instrumental bias estimated from GPS data in solar minimum period summarized in this study (2004) must be smaller than that in solar maximum period. The accuracy of instrumental bias estimated using GPS observations in different solar cycle period was studied initially. It is found that the RMS of the instrumental bias estimated using GPS data observed during solar minimum period is much less than that estimated during solar maximum years (Zhang, 2009b). On the other hand, because the error analysis about the instrumential bias in this study is just based on the results obtained from one estimation methods described in Sect. 2, the quantitative results for the bias error scale given above may not represent the results of other bias estimation methods. Even so, the influence caused by the spatial gradient of the ionosphere should be noted for bias estimation methods based on ionospheric model. 


\section{Summary}

With one bias estimation method, the instrumental biases estimated from the GPS observations in the middle and low latitude region of Chinese longitude sector show obvious differences in the error scale. With the decrease in latitude, the spatial gradient of the ionosphere related to the ionospheric equatorial anomaly increase, and the RMS of the GPS instrumental biases increase. Due to the lower spatial gradient and temporal variation in mid-latitude ionosphere, the satellite and receiver instrumental biases estimated using the same method are more accurate. The RMS values of the satellite instrumental bias estimated from GPS observations in 2004 are around 1 TECU, and the RMS values of the receiver instrumental bias is around 2 TECU in mid-latitude region. Nevertheless, the RMS values of GPS satellite instrumental bias estimated from GPS observations in 2004 near the equatorial anomaly region are around $2 \mathrm{TECU}$, and those of receiver instrumental bias are 3-4 TECU.

Ionospheric TEC derived from GPS data has become one of the routine parameters to describe the ionospheric state in the past two decades. In the process of estimating ionospheric TEC using GPS data, the main error is likely from the estimation of the instrumental bias, so the accuracy of the estimated instrumental bias will directly affect the accuracy of ionospheric TEC. From the analysis above, it can be concluded that the accuracy of the estimated instrumental bias are dependent on the validity of the assumption of the quiet and uniform ionospheric variation condition that must be confronted for any bias estimation methods. Furthermore, the RMS of instrumental bias can be used to estimate the accuracy of TEC based on the GPS method at different latitudes semi-quantitatively. For the estimation method used in this study, the estimated TEC error is around 2-4 TECU based on the mid-latitude GPS observations in 2004, and around 6-8 TECU based on the low latitude GPS observations at the same time.

In any study of the ionospheric morphology or the ionospheric disturbance based on the GPS method, in particular in the study of the weak temporal and spatial variations (such as the ionospheric earthquake effect and the ionospheric influence by meteorological process), the error analysis is needed, to ensure the reliability of results based on ionospheric TEC.

Acknowledgements. We thank CMONOC and IGS for providing highly precise GPS data. This work is jointly supported by the China NSFC (grants: 40674089, 40636032), China NRBRP (grant: 2006CB806306) and State Key Laboratory of Space Weather.

Topical Editor K. Kauristie thanks A. Mazzella and another anonymous referee for their help in evaluating this paper.

\section{References}

Anghel, A., Carrano, C., Komjathy, A., Astilean, A., and Letia, T.: Kalman filter-based algorithms for monitoring the ionosphere and plasmasphere with GPS in near-real time, J. Atmos. Sol.Terr. Phy., 71, 158-174, 2009.

Brunini, C., Meza, A., and Bosch, W.: Temporal and spatial variability of the bias between TOPEX- and GPS-derived total electron content, J. Geodesy, 79, 175-188, doi:10.1007/s00190-0050448-z, 2005.

Carrano, C. S., Anghel, A. F., Quinn, R. A., and Groves, K. M.: Kalman Filter Estimation of Plasmaspheric TEC using GPS, Radio Sci., 44, RS0A10, doi:10.1029/2008RS004070, 2009

Ciraolo, L., Azpilicueta, F., Brunini, C., Meze, A., and Radicella, S. M.: Calibration errors on experimental slant total electron content (TEC) determined with GPS, J. Geodesy, 81, 111-120, doi:10.1007/s00190-006-0093-1, 2007.

Coco, D. S., Coker, C., Dahlke, S. R., and Clynch, J. R.: Variability of GPS satellite differential group delay biases, IEEE T. Aero. Elec. Sys., 27, 931-938, 1991.

Dyrud, L., Jovancevic, A., Brown, A., Wilson, D., and Ganguly, S.: Ionospheric measurement with GPS: Receiver techniques and methods, Radio Sci., 43, RS6002, doi:10.1029/2007RS003770, 2008.

Ho, C. M., Mannucci, A. J., Lindqwister, U. J., Pi, X., Tsurutani, B., et al.: Global ionospheric perturbations monitored by the worldwide GPS network, Geophys. Res. Lett., 23, 3219-3222, 1996.

Ho, C. M., Wilson, B. D., Mannucci, A. J., Lindqwister, U. J., and Yuan, D. N.: A comparative study of ionospheric total electron content measurements using global ionospheric maps of GPS, TOPEX radar, and the Bent model, Radio Sci., 32, 1499-1521, 1997.

Kelley, M. C. and Heelis, R. A.: The Earth's Ionosphere: Plasma Physics and Electrodynamics, Academic Press, 1989.

Lanyi, G. E. and Roth, T.: A comparison of mapped and measured total ionospheric electron content using Global Positioning System and beacon satellites observations, Radio Sci., 23, 483-492, 1988.

Liu, J. Y., Chuo, Y. J., Shan, S. J., Tsai, Y. B., Chen, Y. I., Pulinets, S. A., and Yu, S. B.: Pre-earthquake ionospheric anomalies registered by continuous GPS TEC measurements, Ann. Geophys., 22, 1585-1593, doi:10.5194/angeo-22-1585-2004, 2004.

Lunt, N., Kersley, L., Bishop, G. J., Mazzella, A. J., and Bailey, G. J.: The effect of the protonosphere on the estimation of GPS total electron content: Validation using model simulations, Radio Sci., 34(5), 1261-1271, 1999.

Ma, G. and Maruyama, T.: Derivation of TEC and estimation of instrumental biases from GEONET in Japan, Ann. Geophys., 21, 2083-2093, doi:10.5194/angeo-21-2083-2003, 2003.

Ma, X. F., Maruyama, T., Ma, G., and Takeda, T.: Determination of GPS receiver differential biases by neural network parameter estimation method, Radio Sci., 40(1), RS1002, doi:10.1029/2004RS003072, 2005.

Mannucci, A. J., Wilson, B. D., Yuan, D. N., Ho, C. H., Lindqwister, U. J., and Runge, T. F.: A global mapping technique for GPSderived ionospheric total electron content measurements, Radio Sci., 33, 565-582, 1998.

Mazzella Jr., A. J., Holland, E. A., Andreasen, A. M., Andreasen, C. C., Rao, G. S., and Bishop, G. J.: Autonomous estimation of plasmasphere content using GPS measurements, Radio Sci., 
7(6), 1092, doi:10.1029/2001RS002520, 2002.

Mazzella, A. J., Rao, G. S., Bailey, G. J., Bishop, G. J., and Tsai, L. C.: GPS Determinations of Plasmasphere TEC, Proceedings of the International Beacon Satellite Symposium 2007, edited by: Doherty, P., Boston College, USA, 2007

Mazzella Jr., A. J.: Plasmasphere effects for GPS TEC measurements in North America, Radio Sci., 44, RS5014, doi:10.1029/2009RS004186, 2009.

Mendillo, M.: Storms in the ionosphere: Patterns and processes for total electron content, Rev. Geophys., 44, RG4001, doi:10.1029/ 2005RG000193, 2006.

Niranjan, K., Srivani, B., Gopikrishna, S., and Rama Rao, P. V. S.: Spatial distribution of ionization in the equatorial and low-latitude ionosphere of the Indian sector and its effect on the pierce point altitude for GPS applications during low solar activity periods, J. Geophys. Res., 112(A5), A05304, doi:10.1029/2006JA011989, 2007.

Sardon, E., Rius, A., and Zarraoa, N.: Estimation of the transmitter and receiver differential biases and the ionospheric total electron content from GPS observations, Radio Sci., 29(3), 577-586, 1994.

Sardon, E. and Zarraoa, N.: Estimation of total electron content using GPS data: How stable are the differential satellite and receiver instrumental biases?, Radio Sci., 32, 1899-1910, 1997.

Thampi, Smitha V., Balan, N., Ravindran, Sudha, Pant, Tarun Kumar, Devasia, C. V., Sreelatha, P., Sridharan, R., and Bailey, G. J.: An additional layer in the low-latitude ionosphere in Indian longitudes: Total electron content observations and modeling, J. Geophys. Res., 112(A6), A06301, doi:10.1029/2006JA011974, 2007

Vladimer, J. A., Lee, M. C., Doherty, P. H., Decker, D. T., and Anderson, D. N.: Comparisons of TOPEX and Global Positioning System total electron content measurements at equatorial anomaly latitudes, Radio Sci., 32, 2209-2220, 1997.
Wan, W., Yuan, H., Ning, B., Liang, J., and Ding, F.: Traveling ionospheric disturbances associated with the tropospheric vortexes around Qinghai-Tibet Plateau, Geophys. Res. Lett., 25, 3775-3778, 1998.

Xu, G., Wan, W., She, C., and Du, L.: The relationship between ionospheric total electron content (TEC) over East Asia and the tropospheric circulation around the Qinghai-Tibet Plateau obtained with a partial correlation method, Adv. Space Res., 42, 219-223, 2008.

Zhang, D., Xiao, Z., Gu, S., Ye, Z., and Du, H.: The Study of ionospheric TEC during magnetic storm period on April 6 8, 2000, Journal of Chinese Space Science, 22(3), 220-228, 2002.

Zhang, D. H. and Xiao, Z.: Study of ionospheric response to the 4B flare on 28 October 2003 using International GPS Service network data, J. Geophys. Res., 110, A03307, doi:1029/2004JA010738, 2005.

Zhang, D. H., Xiao, Z., Feng, M., Hao, Y. Q., Shi, L. Q., Yang, G. L., and Suo, Y. C.: The temporal dependence of GPS cycle slip related to ionospheric irregularities over China low latitude region, Space Weather, 8, S04D08, doi:10.1029/2008SW000438, 2010.

Zhang, W., Zhang, D. H., and Xiao, Z.: The influence of geomagnetic storms on the estimation of GPS instrumental biases, Ann. Geophys., 27, 1613-1623, doi:10.5194/angeo-27-16132009, 2009a

Zhang, W.: The influence of ionospheric morphology on the estimation of GPS instrumental biases, Master thesis, Peking Uni, Beijing, 2009b. 